\title{
"Non-criteria" antiphospholipid antibodies add value to antiphospholipid syndrome diagnoses in a large Chinese cohort
}

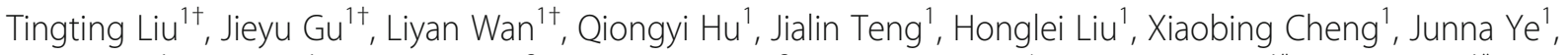
Yutong Su', Yue Sun ${ }^{1}$, Jinfeng Zhou ${ }^{2}$, Gary L. Norman ${ }^{3}$, Xuefeng Wang ${ }^{4}$, Chengde Yang ${ }^{1 *}$ and Hui Shi ${ }^{*}$

\begin{abstract}
Background: Despite expansion in the 2006 Sydney antiphospholipid syndrome (APS) classification criteria to include lgG/lgM anti- 32 -glycoprotein (aß2GPI) antibodies in addition to lgG/lgM anti-cardiolipin antibodies (aCL) and lupus anticoagulant (LAC), some individuals with clinical features of APS remain seronegative (seronegative APS or SNAPS) and are at risk of recurrent thrombosis and pregnancy morbidities. Our aim was to assess the value of "non-criteria" aPL antibodies to detect these SNAPS patients.

Methods: One hundred ninety-two APS patients, 90 SNAPS patients, 193 autoimmune disease controls, and 120 healthy controls were evaluated. Ten antiphospholipid antibodies (aPLs) were tested using commercial kits, including 5 non-criteria aPLs: anti-phosphatidylserine/prothrombin antibodies (aPS/PT) $\operatorname{lgG} / \operatorname{lgM}, \mathrm{aCL} \operatorname{lgA}$, aß2GPI $\lg \mathrm{A}$, and anti-32GPI Domain 1 (aß2GPI-D1) lgG.

Results: Up to $60.9 \%$ of the SNAPS and $93.5 \%$ of APS patients were detected by at least one non-criteria aPL. aPS/ PT IgG had the highest Youden index in classifying APS and SNAPS from controls. aPS/PT IgG and aß2GPI Domain $1 \mathrm{lgG}$ seem to be the most significant risk factors for thrombotic events and pregnancy morbidity, respectively. aPS/ PT IgG/lgM and aß2GPI-D1 lgG were detected in some SNAPS patients, while IgA isotypes of aCL/aß2GPI tended to appear together with other biomarkers. The combined analysis showed enhanced diagnostic performance with the inclusion of non-criteria aPLS.
\end{abstract}

Conclusions: Recognition of SNAPS patients is critical for clinical management and prevention of potential thrombotic and obstetric adverse events. The non-criteria antiphospholipid antibodies help to identify a considerable portion (60.9\%) of these patients who otherwise may remain untreated and at clinical risk.

Keywords: Antiphospholipid syndrome, Non-criteria antiphospholipid antibodies, Anti-phosphatidylserine/ prothrombin antibodies, Anti- $\beta 2$-glycoprotein I Domain 1, IgA isotype

\section{Background}

The antiphospholipid syndrome (APS) is an autoimmune disease characterized by recurrent arterial and/ or venous thrombosis, pregnancy morbidity, and persistent presence of antiphospholipid antibodies (aPLs). APLs have served as important serological markers in the diagnoses of APS since the syndrome was first described

\footnotetext{
*Correspondence: yangchengde@sina.com; shihui_sjtu@sina.com

${ }^{\dagger}$ Tingting Liu, Jieyu Gu and Liyan Wan contributed equally to this work.

'Department of Rheumatology and Immunology, Ruijin Hospital, Shanghai

Jiao Tong University School of Medicine, No. 197 Ruijin Second Road,

Huangpu District, Shanghai 200025, China

Full list of author information is available at the end of the article
}

by Hughes in 1983 [1]. The original APS "Classification Criteria" included only anti-cardiolipin (aCL) IgG/IgM and lupus anticoagulant (LA) as accepted laboratory criteria [2]. In 2006, the sensitivity of the classification criteria was improved by the inclusion of aß2GPI IgG/IgM antibody and the specificity was improved by extending the requirement of persistent presence to 12 weeks [3]. Still, in clinical practice, patients exist with typical clinical manifestations highly suggestive of antiphospholipid syndrome but remain persistently negative for criteria aPLs. These patients have been termed seronegative APS (SNAPS) by Hughs and Khamashta [4]. 
SNAPS patients, just as classic APS patients, manifest increased risk for thrombotic events and pregnancy morbidities. These events may reoccur during the natural course of the disease [5] and in rare cases can result in a life-threatening thrombotic state leading to multi-organ dysfunction known as catastrophic antiphospholipid syndrome (CAPS) [6-8]. Identification of patients with SNAPS, maintaining regular follow-up and providing therapeutic or preventative medication, remains a significant challenge.

Increased recognition and a deeper understanding of APS have evolved with the development of assays for non-criteria aPLs and research into their role in the pathophysiology of APS. These non-criteria aPLs, which include anionic phospholipids, phospholipid-protein complexes, and plasma proteins, may help physicians to better manage suspected APS patients [9]. For example, research on anti-prothrombin $(\mathrm{aPT})$ antibodies eventually led to the recognition of the importance of the complex of phosphatidylserine and prothrombin as a target for aPL antibodies.

Anti-phosphatidylserine/prothrombin antibodies (aPS/ PT) are now acknowledged as a highly effective potential marker for APS $[10,11]$. As a result of its remarkable diagnostic performance and high prevalence in the LA-positive patient group, as validated by multiple studies [12-14], aPS/PT has gained much attention and was included in both the APS-S (Otomo) and the Global APS Score (GAPSS), widely used systems for patient thrombotic risk assessment [15]. Additional biomarkers continue to be evaluated for their value to APS diagnosis and management. The development of assays to specifically examine domainspecific $\beta 2$-glycoprotein antibodies have highlighted the pathogenic role of anti- $\beta 2$ GPI Domain 1 (D1) IgG antibodies [16-18], in contrast to anti-domain 4/5 IgG antibodies which have been characterized as "innocent" antibodies [19]. The IgA isotypes of aPLs are not included as criteria biomarkers; however, they are recommended as adjunctive biomarkers for individuals suspected of APS, but negative for conventional biomarkers [20, 21]. A recent study confirmed the extra predictive value of IgA isotypes when both IgG and IgM were negative [22]. Still, other aPLs, including anti-annexin 5 antibody, anti-protein $\mathrm{C}$ [23], anti-protein S [24], anti-vimentin/cardiolipin complex [25], and anti-lysobisphosphatidic acid (LBPA) [26], also been suggested as relevant new biomarkers, but their value in APS diagnosis and management is still unclear. In the last few years, new methodological approaches, including thin-layer chromatography (TLC) [27, 28], multiline dot assay [29], and chemiluminescence [30], have been developed for refining laboratory diagnosis of APS. The antigenic presentation of proteins and/or phospholipid/protein complexes are quite different in these three systems as compared to standard ELISA, and they might be useful to expand our knowledge on the antigen specificities of "antiphospholipid antibodies."

In the current study, five of the most promising noncriteria antibodies for which commercial kits were widely available, namely aCL IgA, aß2GPI IgA, aPS/PT IgG/IgM, anti- $\beta 2$ GPI Domain 1 (aß2GPI D1) IgG, were selected for evaluation. The value of these markers to assist in the detection of SNAPS patients and their value in predicting in adverse clinical events as assessed by odds ratio (OR) were analyzed in a large cohort of APS and seronegative patients, as well as in patients with a variety of other autoimmune diseases and healthy adults as controls. The individual and the combined value of the non-criteria antibodies in classifying APS, as well as SNAPS patients, were then evaluated.

\section{Methods}

\section{Patients recruitment}

A total of 595 patients were included in this case-control study. The research was performed according to the Declaration of Helsinki and approved by the Institutional Review Broad of Ruijin Hospital (ID: 2016-62), Shanghai Jiaotong University School of Medicine, Shanghai, China. Informed consent was obtained from all individuals included in this study. Patients were classified into seven subgroups:

-Group 1: 192 APS patients from the APS-Shanghai (APS-SH) database, which was established by expert rheumatologists and statisticians at the Shanghai Jiao Tong University School of Medicine (Shanghai, China). All patients satisfied the 2006 Sydney classification criteria. In this group, 88 patients were classified as primary antiphospholipid syndrome (PAPS) and 104 patients as secondary antiphospholipid syndrome (SAPS) patients, of which 76 were comorbid with SLE, 25 with lupus-like disease, 2 with Sjogren's syndrome, and 1 with rheumatoid arthritis.

-Group 2: 90 SNAPS patients from the APS-SH database fulfilling the Sydney clinical criteria but persistently negative for criteria aPLs. Diagnoses were established and confirmed by at least two expert rheumatologists in accordance with the definition by Hughs and Khamashta [4], and hereditary and other acquired thrombophilia were excluded before patients were enrolled. In addition to the major clinical criteria that fulfilled the Sydney classification criteria for APS, at least one of the noncriteria manifestations associated with APS were also required to make the diagnosis of SNAPS. The noncriteria clinical features are as follows: (1) superficial vein thrombosis, (2) thrombocytopenia, (3) renal microangiopathy, (4) heart valvular disease, (5) livedo reticularis, (6) migraine, (7) chorea, (8) seizure, (9) longitudinal myelitis, (10) epilepsy, (11) Raynaud's phenomenon, and (12) brain MRI showed white matter 
lesions [5, 31]. More details about this group of patients were added in the text as thrombocytopenia was recorded in 33.33\% (30/90) SNAPS. A history of heart valvular disease and livedo reticularis were frequently documented in SNAPS (21.11\% (19/90) and 17.78\% (16/ 90), respectively). There are $16.67 \%$ (15/90) patients with brain MRI showed white matter lesions. 8.89\% (8/90) of patients in the SNAPS group developed Raynaud's phenomenon. Migraine was found in $7.78 \%$ (7/90) SNAPS patients. And epilepsy is recorded in $4.44 \%$ (4/ 90) patients. There are 9 patients with two of these manifestations.

-Group 3: 103 patients with systemic lupus erythematosus (SLE) satisfying both the revised 1997 American College of Rheumatology(ACR) set of classification criteria [32] and the 2012 criteria established by the Systemic Lupus International Collaborating Clinics (SLICC) group [33].

-Group 4: 29 patients with Sjogren's syndrome (SS) satisfying the ACR/EULAR 2016 classification criteria [34].

-Group 5: 30 patients with ankylosing spondylitis (AS) satisfying the 1984 revised New York Criteria [35].

-Group 6: 31 patients with rheumatoid arthritis (RA) satisfying ACR/EULAR 2010 classification criteria [36].

-Group 7: 120 healthy controls (HC), without autoimmune, neoplastic or infectious diseases.

Demographic characteristics of the cohort are depicted in Table 1. All patients were Chinese and were enrolled continuously from 2000 to 2017. Complete medical histories, laboratory tests, and medical images were recorded in the database and all patients underwent regular follow-up either by the outpatient department or by telephone follow-up. Thrombotic events and pregnancy morbidities (PM) were recorded. Thrombosis was classified into arterial thrombosis and venous thrombosis, while pregnancy morbidities were divided into early and late pregnancy. Early pregnancy morbidity referred to at least one fetal loss before 10 weeks of pregnancy while late pregnancy morbidity referred to at least one stillbirth after 10 weeks of pregnancy or premature delivery before 34 weeks of pregnancy. The occurrence of stroke and deep vein thrombosis was also recorded. Serum samples were collected and frozen at $-80^{\circ} \mathrm{C}$ until testing. Diagnoses were confirmed by at least two rheumatologists from Shanghai Jiao Tong University School of Medicine. Serum levels of the criteria aPLs, except for lupus anticoagulants, were determined by semiquantitative QUANTA Lite $^{\circ}$ ELISA kits provided by Inova Diagnostics, Inc. (San Diego, CA, USA). Lupus anticoagulant was detected and evaluated by the clinical laboratory of Ruijin Hospital according to the ISTH recommendations. The assay was performed at the patient's first visit to the rheumatology department and repeated 12 weeks later.

\section{Laboratory tests \\ Anti-cardiolipin antibodies (aCL) and anti- $\beta 2$-glycoprotein I antibodies (aß2GPI)}

aCL IgG/IgM/IgA and aß2GPI IgG/IgM/IgA were measured using QUANTA Flash ${ }^{\circ}$ (Inova Diagnostics Inc., San Diego, CA, USA) chemiluminescent immunoassays run on the BIO-FLASH ${ }^{\circ}$ instrument (Biokit s.a., Barcelona, Spain). The cutoff values for the antibodies were defined as 20 chemiluminescent units $(\mathrm{CU})$ as recommended by the manufacturer.

Table 1 Demographic characteristics and antibody profile of APS and SNAPS patients

\begin{tabular}{|c|c|c|c|c|c|c|c|}
\hline & APS & SNAPS & SLE & SS & RA & AS & $\mathrm{HC}$ \\
\hline Numbers & 192 & 90 & 103 & 29 & 31 & 30 & 120 \\
\hline Age (Q1-Q3) & $35(30-46)$ & $39(32-51)$ & $34(29-43)$ & $42(35-51)$ & $47(40-58)$ & $34.5(27-40)$ & $39(34-46)$ \\
\hline $\operatorname{Sex}(F / M)$ & $162 / 30$ & $77 / 13$ & $92 / 8$ & $27 / 2$ & $28 / 3$ & $15 / 15$ & $108 / 12$ \\
\hline Arterial thrombosis & $74(39 \%)$ & $53(59 \%)$ & $0(0 \%)$ & $0(0 \%)$ & $0(0 \%)$ & $0(0 \%)$ & $0(0 \%)$ \\
\hline Venous thrombosis & 78 (41\%) & $33(37 \%)$ & $0(0 \%)$ & $0(0 \%)$ & $0(0 \%)$ & $0(0 \%)$ & $0(0 \%)$ \\
\hline Early fetal loss & $36(22 \%)$ & $12(16 \%)$ & $0(0 \%)$ & $0(0 \%)$ & $0(0 \%)$ & $0(0 \%)$ & $0(0 \%)$ \\
\hline Late fetal loss & 81 (50\%) & $18(23 \%)$ & $0(0 \%)$ & $0(0 \%)$ & $0(0 \%)$ & $0(0 \%)$ & $0(0 \%)$ \\
\hline $\mathrm{aCL} \lg G / \lg M(+)$ & $115(60 \%)$ & $0(0 \%)$ & $6(6 \%)$ & $0(0 \%)$ & $0(0 \%)$ & $0(0 \%)$ & $0(0 \%)$ \\
\hline aB2GPI lgG/lgM(+) & $130(68 \%)$ & $0(0 \%)$ & $13(13 \%)$ & $0(0 \%)$ & $0(0 \%)$ & $1(3 \%)$ & $0(0 \%)$ \\
\hline $\mathrm{LA}(+)$ & 133 (69\%) & $0(0 \%)$ & $6(6 \%)$ & $0(0 \%)$ & $0(0 \%)$ & $0(0 \%)$ & $0(0 \%)$ \\
\hline $\mathrm{aCL} \lg \mathrm{A}(+)$ & $80(42 \%)$ & $11(12 \%)$ & $12(12 \%)$ & $0(0 \%)$ & $0(0 \%)$ & $0(0 \%)$ & $0(0 \%)$ \\
\hline aB2GPI $\operatorname{lgA}(+)$ & 75 (39\%) & $9(10 \%)$ & $7(7 \%)$ & $0(0 \%)$ & $0(0 \%)$ & $0(0 \%)$ & $0(0 \%)$ \\
\hline aPS/PT IgG(+) & 137 (71\%) & $32(36 \%)$ & 15 (15\%) & $1(3 \%)$ & $0(0 \%)$ & $0(0 \%)$ & $0(0 \%)$ \\
\hline aPS/PT IgM(+) & 141 (73\%) & $32(36 \%)$ & $33(32 \%)$ & 7 (24\%) & $0(0 \%)$ & $3(10 \%)$ & $5(4 \%)$ \\
\hline aB2GPI D1 lgG(+) & 119 (62\%) & 14 (16\%) & $7(7 \%)$ & $0(0 \%)$ & $0(0 \%)$ & $0(0 \%)$ & $2(2 \%)$ \\
\hline
\end{tabular}


Anti-phosphatidylserine-prothrombin antibodies (aPS/PT) Anti-PS/PT IgG and IgM antibodies were measured using semi-quantitative QUANTA Lite ${ }^{\bullet}$ ELISA kits (Inova Diagnostics Inc., San Diego, CA, USA). The cutoff values for both aPS/PT IgG and IgM were defined as 30 units $(\mathrm{U})$ as recommended by the manufacturer.

\section{Anti- $\beta 2$-glycoprotein I Domain 1 antibodies (aß2GPI D1)} aß2GPI D1 IgG was measured using QUANTA Flash ${ }^{\circ}$ (Inova Diagnostics Inc., San Diego, CA, USA) chemiluminescent immunoassay. The cutoff value was $\geq 20 \mathrm{CU}$ as defined by the manufacturer.

\section{Statistical analysis}

Statistical analysis was performed using R (version 3.5.1). In descriptive statistics, data were expressed in the form of positive numbers, percentages for categorical variables, and median (Q1-Q3) for continuous variables. Chi-Squared test and Fisher's exact were used to compare the categorical variables, and the Wilcoxon test and Kruskal-Wallis test were used to compare the continuous variables after normality was explored with the Shapiro-Wilk test. Logistic regression models with adjusted gender and age information were used to calculate the odds ratios between different serum markers and clinical events. The outcome of the ORs is presented together with the 95\% Wilson confidence interval (CI). The level of statistical significance was set at a two-tailed $\alpha$-value of 0.05 by default. Diagnostic performances of the different biomarkers were evaluated by calculating the sensitivity, specificity, positive predictive value (PPV), negative predictive value (NPV), positive likelihood ratio (PLR), and negative likelihood ratio (NLR). Both the APS and SNAPS groups are considered the experimental group when evaluating the diagnostic indexes using fourfold table. Relationships between aPL profiles and clinical events are presented as odds ratios.

\section{Results}

\section{Distribution of antiphospholipid antibodies}

Distributions of the nine different criteria and noncriteria antibodies among the different clinical groups are portrayed in Fig. 1, and the frequencies of occurrence in is tabulated in Table 1 . The serum levels of different aPLs are significantly elevated in the classic APS

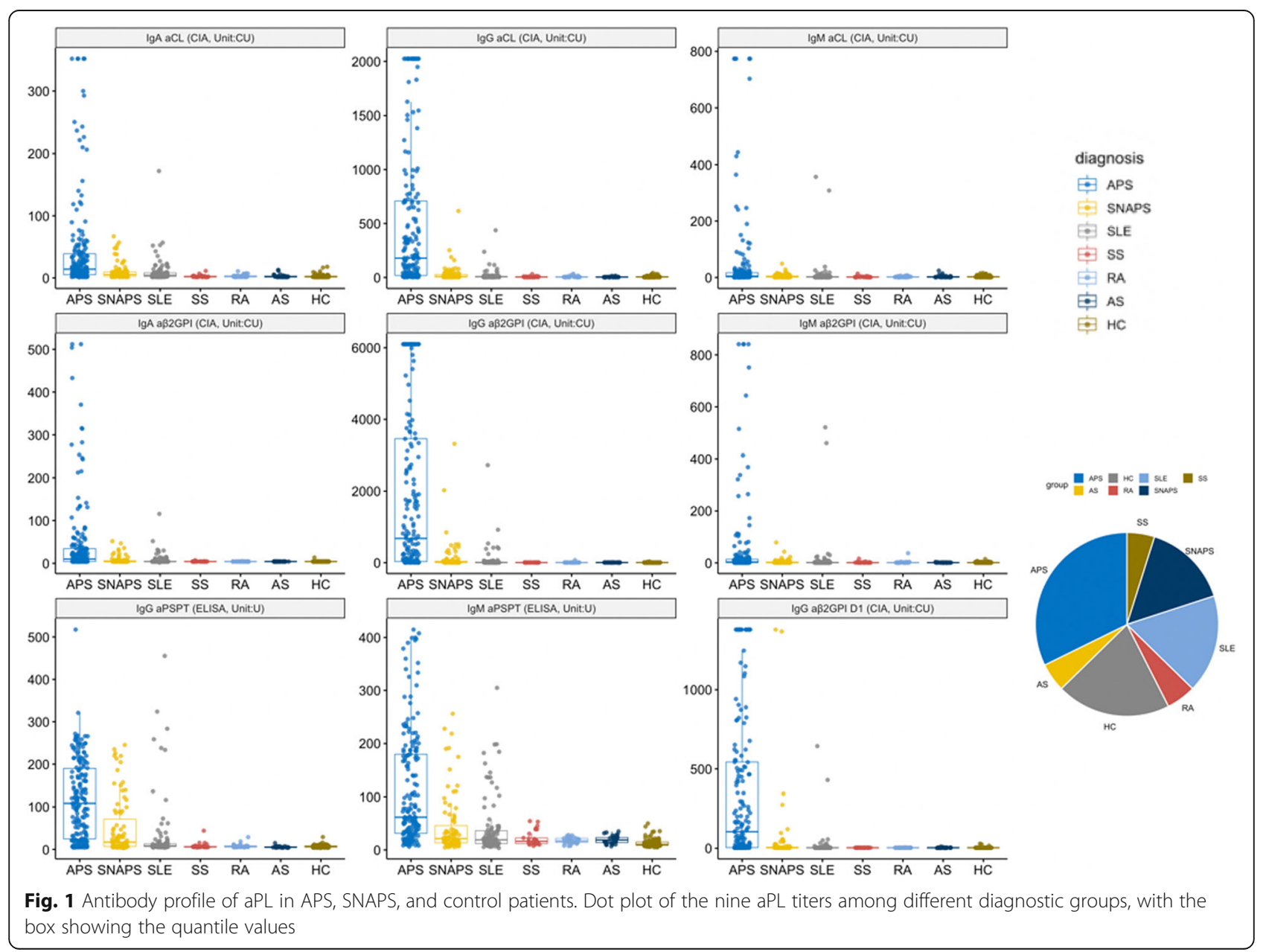


patients, while patients with SNAPS and SLE also have increased antibody levels compared with other disease controls or healthy controls. IgG isotypes of the antibodies show better reproducibility in the disease group and are more uniformly distributed compared with the IgA and IgM isotypes. The prevalence and titer of various aPL among the different subset of patients (SNAPS/single/ double/triple positive) are illustrated in Fig. 2. Diagnostic values of the aPLs in APS and SNAPS patients were assessed by receiver operating characteristic curve (ROC) analysis (Fig. 3). Anti- $\beta 2$ GPI IgG showed the largest area under the curve (AUC, 0.875), followed by aPS/PT IgG (AUC, 0.836) and aCL IgG (AUC, 0.836).

\section{Prevalence of non-criteria aPLs in SNAPS group}

The complementary ability of the non-criteria aPLs to identify SNAPS patients is among the most important aspects in the study of new antibodies. Figure 4 shows the number (from the set of aCL IgA, aß2GPI IgA, aPS/ PT IgG, aPS/PT IgM, and aß2GPI D1 IgG) of positive non-criteria aPLs among the different clinical groups.
93.5\% of the APS patients have at least one positive noncriteria antibody, a majority of these groups have three or more positive non-criteria antibodies, and $23.4 \%$ APS patients are positive for all five non-criteria aPLs. In the SNAPS and SLE groups, $60.9 \%$ and $40.8 \%$ of patients respectively are detected by non-criteria aPLs.

Additional analysis (Fig. 5) demonstrated that aPS/PT IgG and aPS/PT IgM are the most frequently detected aPLs in both APS and SNAPS patients. Also, the prevalence of aß2GPI D1 IgG is higher in the SNAPS group than in the SLE group. It is important to note that aPS/ PT IgG, aPS/PT IgM, and aß2GPI D1 IgG can each be the only biomarkers detected in SNAPS patients, in contrast to aCL IgA and a 32 GPI IgA antibodies which tend to appear accompanied by other non-criteria aPLs.

\section{Relationship between clinical manifestations and criteria as well as non-criteria aPLs}

To explore whether the non-criteria aPLs could add value for the prognostic prediction of clinical events, a radar plot (Fig. 6) depicting the odds ratios for clinical

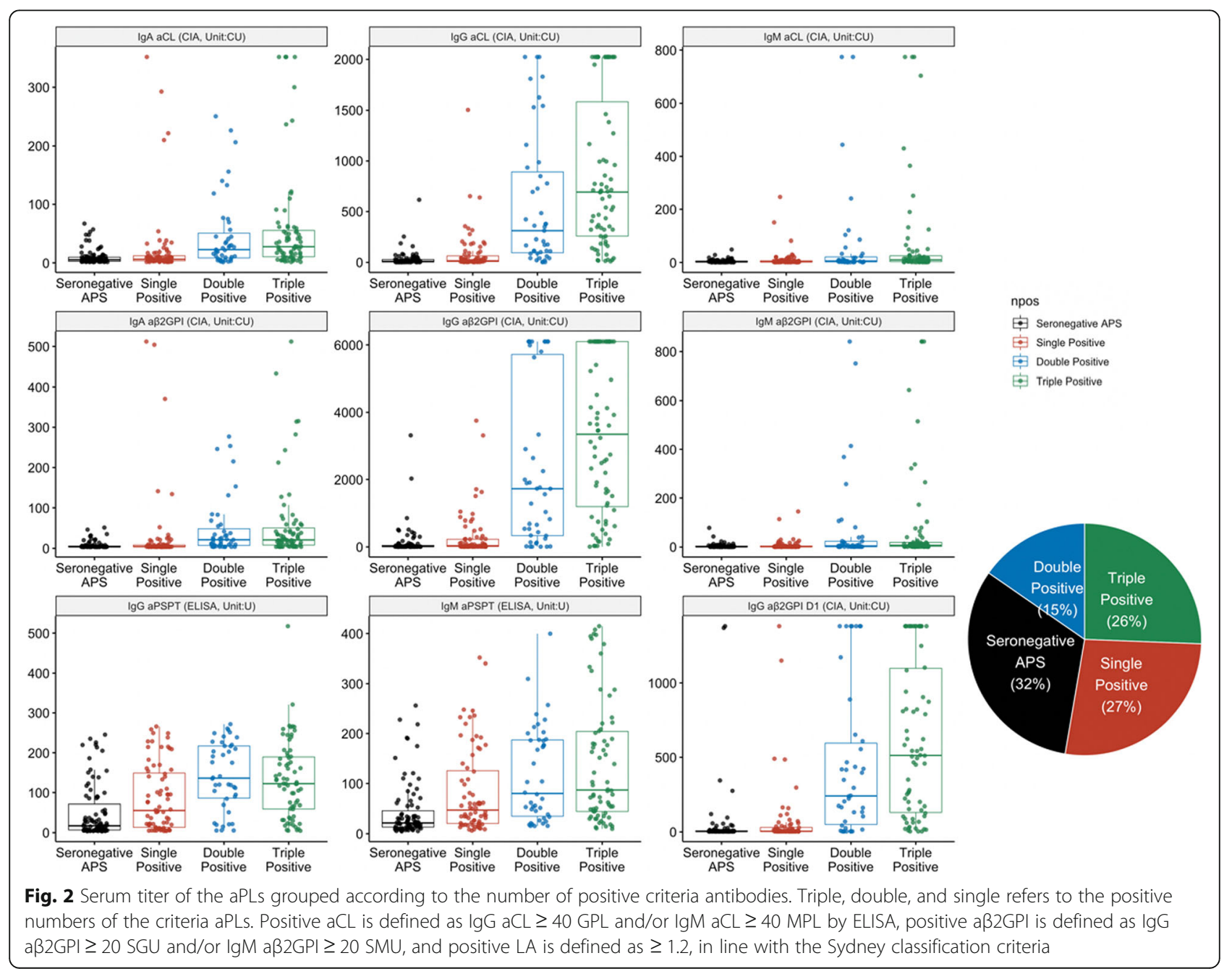




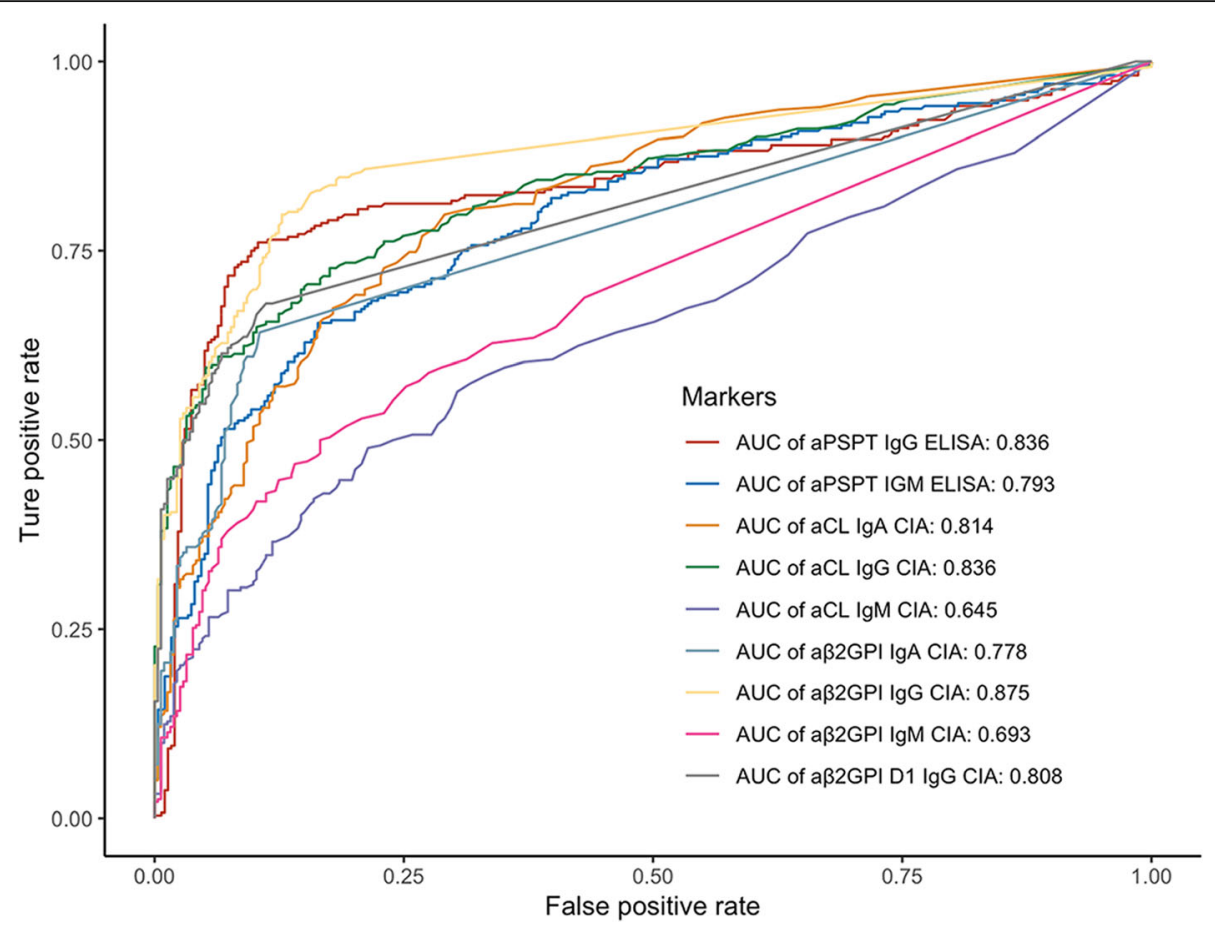

Fig. 3 ROC plot of the individual aPLs

events was constructed. As can be seen from the figure, aß2GPI IgG is the best predictor of arterial thrombosis with the odds ratio of 6.5 (95\% CI, 3.64-8.75), while aPS/PT IgG with an odds ratio of 7.46 (95\% CI, 4.6911.88 ) is closely associated with venous thrombosis.
Interestingly, stroke can be best predicted by aß2GPI IgG, with the odds ratio of 5.01 (95\% CI, 2.91-8.6), while aPS/PT IgG best predicts deep vein thrombosis with the odds ratio of 9.02 (95\% CI, 5.5-14.81). With regards to early pregnancy morbidity, aPS/PT IgM shows the best

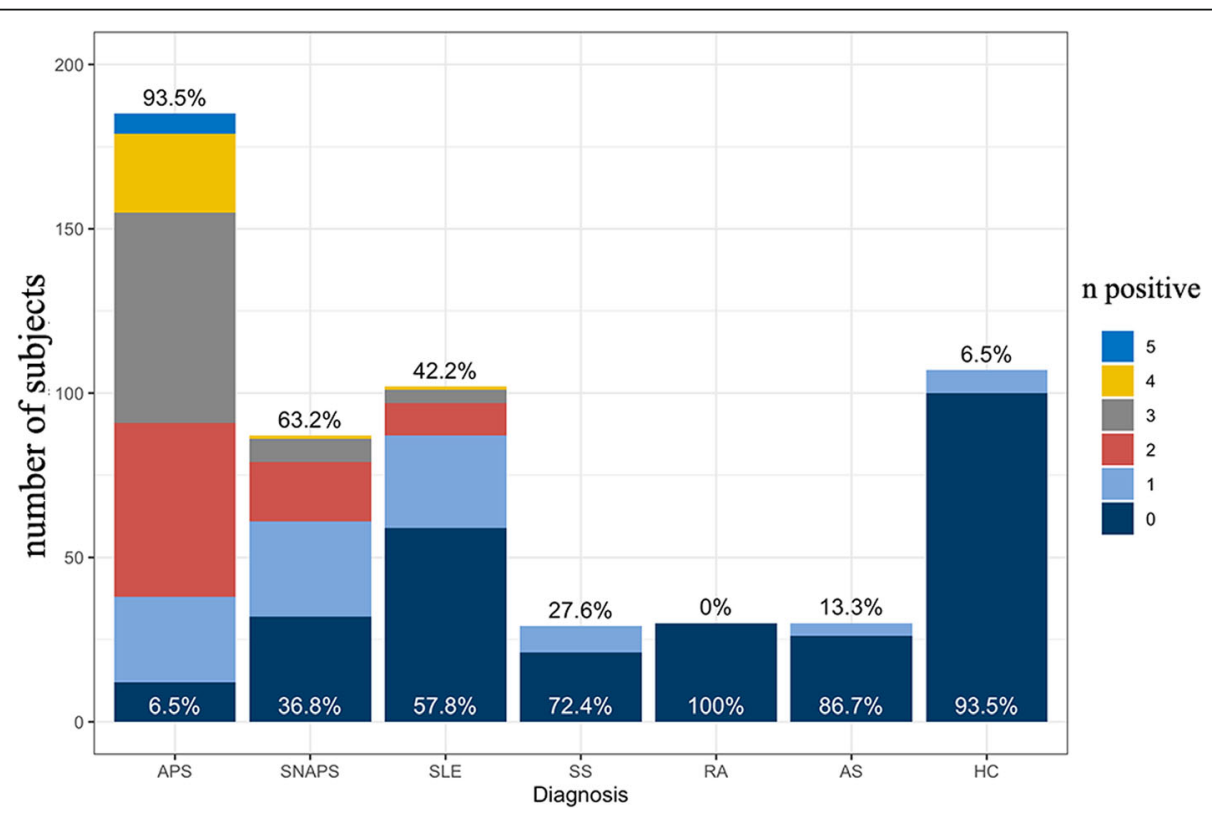

Fig. 4 The number of the five non-criteria aPLs in different clinical groups. The black percentage above the bars indicates the percentage of patients with any positive non-criteria antibodies, while the white percentage on the bottom indicates those without positive non-criteria antibodies 


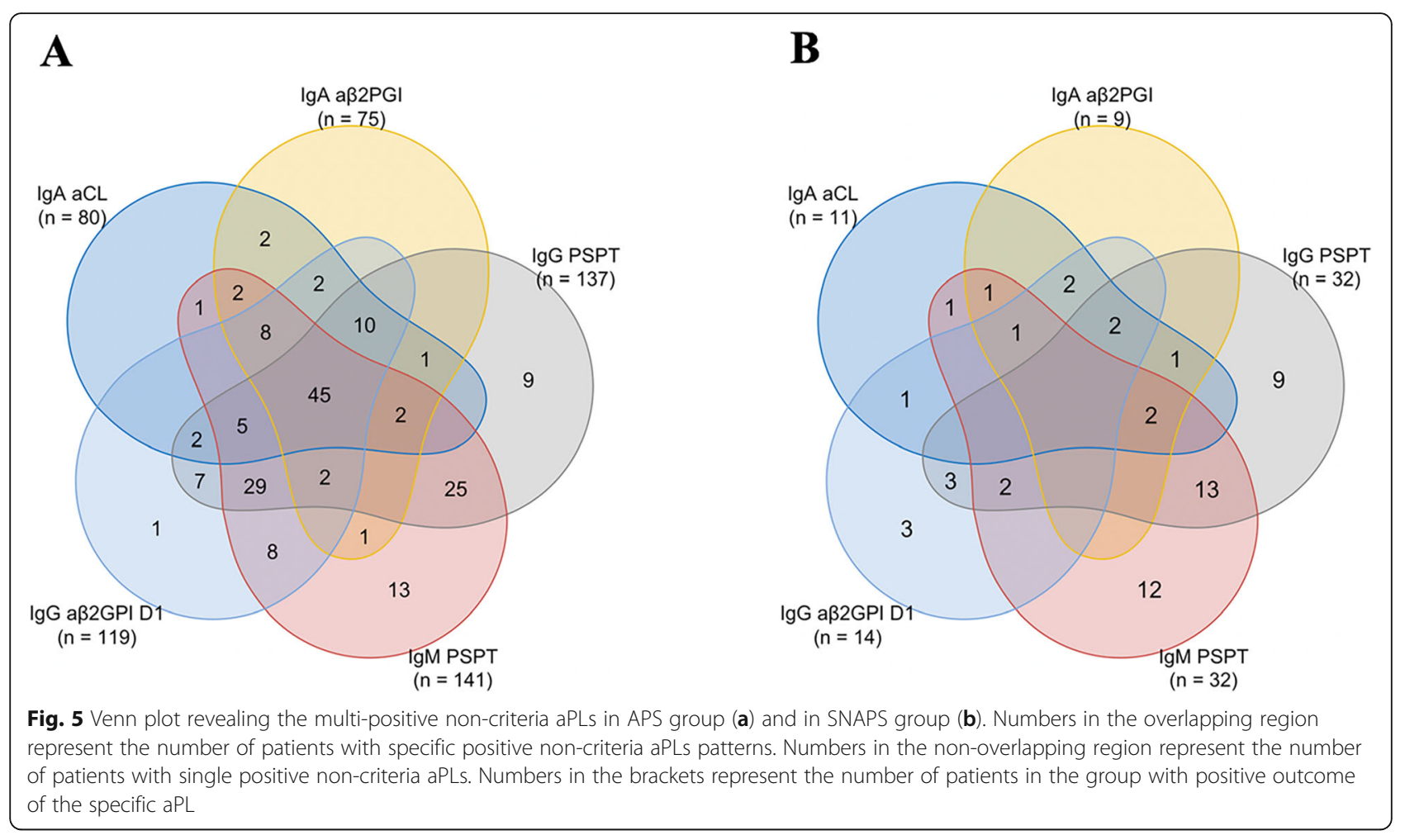

association with an odds ratio of 3.78 (95\% CI, 1.947.36). On the other hand, a $\beta 2$ GPI IgG best predicts late pregnancy morbidity with an odds ratio of 10.21 (95\% CI, 6.03-17.27). None of the odds ratios for the various non-criteria biomarkers are less than 1 in the analysis. Regardless of the detailed clinical manifestations, aPS/ PT IgG and aß2GPI Domain 1 IgG seem to be the most significant risk factors for thrombotic events and pregnancy morbidity, respectively.

From another perspective, IgG isotypes of aCL and a $\beta 2$ GPI are better risk factors for the clinical manifestations of APS, followed by IgA aCL/aß2GPI, while the IgM isotypes of the same aPL show the least association with the APS-related clinical events.

\section{Individual and combined values of the criteria and non- criteria aPLs in diagnosing APS and SNAPS patients}

All combinations of the 10 biomarkers were enumerated and to find the best combinations of the aPLs, including LAC, to best define these groups of patients. Combinations of the biomarkers in all are analyzed, including 10 single markers. The performance of different aPLs was scored according to the Youden index and the OR. The results of the individual biomarkers and part of the combinations are shown in Table 2 .

As to the single biomarkers, a 32 GPI IgG had the best sensitivity of 0.6702 , while the best specificity belongs to LAC with a value of 0.9808 in the current cohort. Overall, aPS/PT IgG appears to be a more balanced biomarker with the max Youden index of 1.5678 (sensitivity and specificity being 0.6312 and 0.9465 , respectively). aß2GPI Domain 1 IgG, which has been recognized as a pathogenic antibody of APS, has better specificity (0.9712) but much lower sensitivity (0.489) compared with the IgG antibody to the whole $\beta 2 \mathrm{GPI}$ molecule. aCL/aß2GPI IgA possess nearly twice the sensitivity compared to the IgM isotypes of aCL/aß2GPI, while maintaining similar specificity. Isolated lupus anticoagulant positivity has the highest OR of 45.67 (95\% CI, 19.7-105.9), for the diagnosis of APS.

As to the combined analysis, the aPL combination with the largest Youden index is the combination of aPS/PT IgG, aCL IgA, a $\beta 2$ GPI IgA, aß2GPI IgG, and LAC, with a sensitivity and specificity of $84.93 \%$ and 87.63\%, respectively, and an OR of 39.9 (95\% CI, 24.7364.37). The combination of aPS/PT IgG, aCL IgG, and LAC provides outstanding performance with a sensitivity of $82.35 \%$ and specificity of $89.97 \%$ with the least number of biomarkers.

In the next step, we rank the combinations according to the Youden indexes from largest to smallest and displayed the $10 \mathrm{aPL}$ combinations with the biggest Youden indexes in Table 2. The frequency of occurrence of single biomarkers was added up for the sake of reproducibility. Anti-PS/PT IgG and LAC were present in all the combinations, supporting the robustness of the clinical value contributed by these two markers, followed by aß2GPI IgG with an occurrence frequency of 0.9 , and 


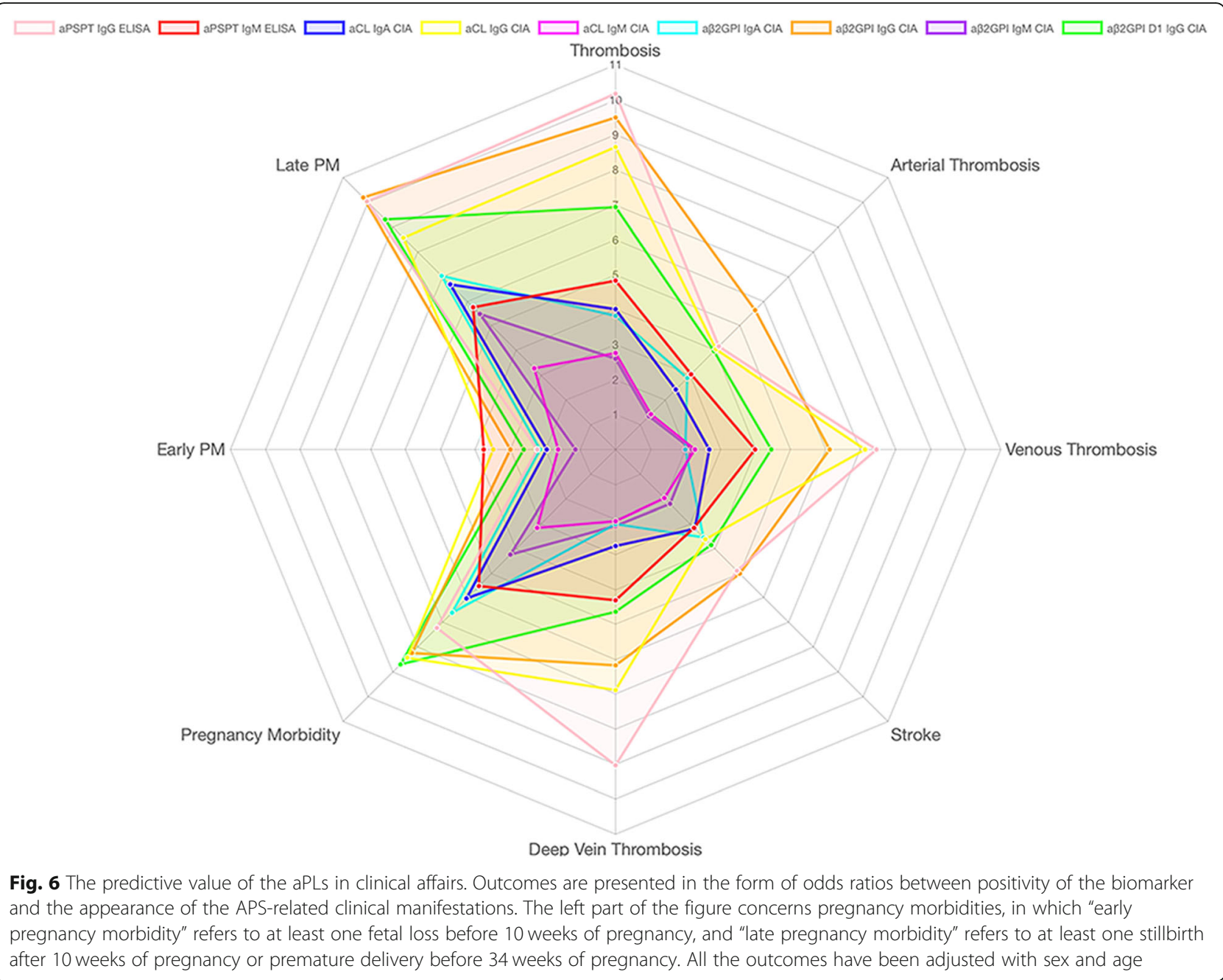

then IgA a $\beta 2$ GPI and IgA aCL. Interestingly, aCL IgG is less often included in the best combinations, perhaps because of its strong correlation with other pathogenic antibodies.

\section{Discussion}

While it has long been accepted that aPLs contribute to the pathogenesis of APS, the discordance between the persistent presence of aPLs (which may not fluctuate upon medical intervention) and isolated clinical adverse events has remained a continuous area of research focus. Discoveries of new aPLs have helped detect potential seronegative patients as well as provide additional insight into the mechanisms of the disease. Although there is a vast array of aPL biomarkers which have shown some indication of clinical or diagnostic value, most have not been replicated or well-validated outside of the research lab. In the present study, we chose five non-criteria aPLs, namely the IgA isotypes of aCL and a 32 GPI, aPS/PT IgG and IgM, and anti- $\beta 2$ GPI Domain 1 IgG (aß2GPI D1 IgG) antibody. All five of these non- criteria aPLs can be detected in a considerable proportion of the SNAPS patients and could help physicians to identify patients with an elevated risk of thrombosis and pregnancy morbidity who may otherwise be missed.

Concerning the individual biomarkers, although the value of the IgA isotypes of the aPLs has been questioned because of varying prevalence among different study populations [37] and the heterogeneity between different assays [38], in the present study, the IgA isotypes of $\mathrm{aCL} / \mathrm{a} \beta 2 \mathrm{GPI}$ far exceeded the IgM isotypes in sensitivity while maintaining very high specificity. Furthermore, the presence of anti-aPL IgA antibodies was related to a higher risk for thrombosis and pregnancy morbidity than the IgM aPLs. Although the prevalence of IgA aCL and IgA a 2 GPI was lower than the other non-criteria aPLs in both the APS and SNAPS group in our cohort, these markers can also provide additional hints for SNAPS diagnosis when other laboratory tests are unclear.

Several previous studies have suggested aPS/PT antibodies might be used as a surrogate for lupus 
Table 2 Diagnostic values of the criteria and non-criteria aPLs

\begin{tabular}{|c|c|c|c|c|c|c|c|c|}
\hline Parameters & Sen & Spe & PPV & NPV & PLR & NLR & OR $(95 \% \mathrm{Cl})$ & Youden index \\
\hline LAC & 0.4716 & 0.9808 & 0.9568 & 0.6732 & 24.6034 & 0.5387 & 45.67 (19.7-105.9) & 1.4524 \\
\hline $\mathrm{aCL} \lg \mathrm{A}$ & 0.3227 & 0.9617 & 0.8835 & 0.6118 & 8.417 & 0.7043 & $11.95(6.37-22.41)$ & 1.2844 \\
\hline $\mathrm{aCL} \lg G$ & 0.5993 & 0.9425 & 0.9037 & 0.723 & 10.421 & 0.4252 & $24.51(14.4-41.73)$ & 1.5418 \\
\hline $\mathrm{aCL} \lg \mathrm{M}$ & 0.1525 & 0.9808 & 0.8776 & 0.5623 & 7.9545 & 0.8641 & $9.21(3.85-21.99)$ & 1.1333 \\
\hline $\mathrm{aCL} \lg \mathrm{G} / \lg M$ & 0.6277 & 0.9265 & 0.885 & 0.7342 & 8.5416 & 0.4019 & $21.25(13.04-34.64)$ & 1.5542 \\
\hline $\mathrm{aCL} \lg \mathrm{A} / \mathrm{G} / \mathrm{M}$ & 0.6418 & 0.9105 & 0.866 & 0.7383 & 7.1749 & 0.3933 & $18.24(11.54-28.84)$ & 1.5523 \\
\hline $\mathrm{a} \beta 2 \mathrm{GPI} \lg \mathrm{A}$ & 0.2979 & 0.9776 & 0.9231 & 0.6071 & 13.3191 & 0.7182 & 18.55 (8.4-40.92) & 1.2755 \\
\hline $\mathrm{a} \beta 2 \mathrm{GPI} \lg \mathrm{G}$ & 0.6702 & 0.9137 & 0.875 & 0.7546 & 7.7695 & 0.3609 & $21.53(13.51-34.31)$ & 1.5839 \\
\hline aß2GPI IgM & 0.1383 & 0.9776 & 0.8478 & 0.5574 & 6.1839 & 0.8814 & $7.02(3.08-15.96)$ & 1.1159 \\
\hline aß2GPI D1 & 0.489 & 0.9712 & 0.9366 & 0.6862 & 17.0053 & 0.5262 & $32.32(15.98-65.36)$ & 1.4602 \\
\hline $\mathrm{a} \beta 2 \mathrm{GPI} \lg G / \mathrm{M}$ & 0.6879 & 0.9073 & 0.87 & 0.7634 & 7.425 & 0.3439 & $21.59(13.66-34.12)$ & 1.5952 \\
\hline aB2GPI $\lg A / G / M$ & 0.695 & 0.9073 & 0.8711 & 0.7676 & 7.5016 & 0.3361 & $22.32(14.11-35.3)$ & 1.6023 \\
\hline aB2GPI lgG/M/D1 & 0.6949 & 0.9042 & 0.863 & 0.7732 & 7.2496 & 0.3375 & $21.48(13.61-33.9)$ & 1.5991 \\
\hline $\mathrm{aB} 2 \mathrm{GPI} \lg \mathrm{A} / \mathrm{G} / \mathrm{M} / \mathrm{D} 1$ & 0.7022 & 0.9042 & 0.8643 & 0.7775 & 7.3263 & 0.3294 & $22.24(14.08-35.14)$ & 1.6064 \\
\hline aPSPT IgG & 0.6213 & 0.9465 & 0.9135 & 0.7332 & 11.611 & 0.4001 & $29.02(16.58-50.81)$ & 1.5678 \\
\hline aPSPT IgM & 0.636 & 0.8395 & 0.7828 & 0.7171 & 3.9619 & 0.4336 & $9.14(6.15-13.57)$ & 1.4755 \\
\hline aPSPT IgG/M & 0.7978 & 0.8127 & 0.7949 & 0.8154 & 4.2597 & 0.2488 & $17.12(11.31-25.92)$ & 1.6105 \\
\hline $\mathrm{aCL} \operatorname{lgG} / \mathrm{M} / \mathrm{aB} 2 \mathrm{GPI} \lg \mathrm{G} / \mathrm{M} / \mathrm{LAC}$ & 0.7872 & 0.8626 & 0.8377 & 0.8182 & 5.7303 & 0.2467 & $23.23(15.11-35.71)$ & 1.6498 \\
\hline $\mathrm{aCL} \lg \mathrm{A} / \mathrm{G} / \mathrm{M} / \mathrm{aB} 2 \mathrm{GPI} \lg \mathrm{A} / \mathrm{G} / \mathrm{M} / \mathrm{LAC}$ & 0.7943 & 0.8594 & 0.8358 & 0.8226 & 5.6505 & 0.2393 & $23.61(15.36-36.3)$ & 1.6537 \\
\hline $\mathrm{aCL} \operatorname{lgG} / \mathrm{M} / \mathrm{aB} 2 \mathrm{GPI} \operatorname{lgG} / \mathrm{M} / \mathrm{D} 1 / \mathrm{LAC}$ & 0.7831 & 0.8594 & 0.8288 & 0.8201 & 5.5706 & 0.2524 & $22.07(14.36-33.92)$ & 1.6425 \\
\hline aPSPT IgG/M/aCL IgG/M/aB2GPI lgG/M/LAC & 0.8897 & 0.7592 & 0.7707 & 0.8833 & 3.6948 & 0.1453 & $25.43(16.01-40.4)$ & 1.6489 \\
\hline aPSPT IgG/aCL IgA/aB2GPI IgA/G/LAC & 0.8493 & 0.8763 & 0.8619 & 0.8647 & 6.863 & 0.172 & $39.9(24.73-64.37)$ & 1.7256 \\
\hline aPSPT IgG/aCL IgG/LAC & 0.8235 & 0.8997 & 0.8819 & 0.8486 & 8.2078 & 0.1962 & $41.84(25.65-68.26)$ & 1.7232 \\
\hline
\end{tabular}

anticoagulant detection [14] since the latter assay and its ancillary confirmatory assays is technically demanding and is not performed in many laboratories. Lupus anticoagulant remains a mysterious set of heterogeneous antibodies yet to be confirmed, while aPS/PT antibodies stand for an independent biomarker for APS and add value to SNAPS patients' management.

Antibodies to $\beta 2 \mathrm{GPI}$-D1, which are a subset of antibodies to the whole $\beta 2$ GPI molecule, showed a close relationship with pregnancy morbidities, especially with late pregnancy morbidity in our cohort, which is in accordance with a recently published retrospective study of obstetric patients [39].

In the combined analysis of the aPLs, adding the noncriteria aPLs separately or together added to the sensitivity of the total assay but simultaneously sacrificed the specificity, so maybe "the more the better" is not necessarily true in this case. Thus, in this study, the Youden index and odds ratio were used to evaluate the diagnostic performance of the antibody profiles. There is increasing recognition that scoring systems developed to quantify the contribution of different biomarkers and clinical manifestations (including the criteria and extracriteria manifestations), as has been done in other rheumatic diseases. It is useful to evaluate and manage APS patients with the APS score and the Global APS Score in mind.

As to the relationship between aPLs and clinical manifestations, all of the biomarkers explored here are better predictors of late pregnancy morbidity rather than early pregnancy morbidity, supporting the concept that late pregnancy morbidity is a more specific clinical manifestation of the disease. Our results showed a higher prevalence of non-criteria aPL compared with similar studies previously reported $[38,40,41]$. On the one hand, variability between commercial diagnostic kits and lack of standardization among laboratories may account for the discrepancy. On the other hand, it perhaps reflects the specific makeup of our very well-characterized clinical cohort.

This study has some limitations. Histories of further clinical manifestations such as livedo reticularis, thrombocytopenia, valvular heart disease, epilepsy, and other manifestations related to aPLs may be included to better perform the analysis. As a result of the retrospective nature of our study, whether these aPL-positive patients will develop into APS is unknown and will be examined in a subsequent study. 
APS can occur as an isolated diagnosis (primary APS), or it can be associated with systemic lupus erythematosus (SLE) and some of the criteria aPLs were included in the 1997 classification criteria for SLE of the American College of Rheumatology [32]. In clinical practice, APS is seen far more frequently in SLE or lupus-like disease than other autoimmune diseases. In the present study, a considerable proportion of the patients suffer APS secondary to SLE. On the other hand, some patients develop SLE several years after the manifestations of APS appear [42]; thus, while the relationship between APS and SLE is close, the factors leading to one condition or the other remain unclear. Close observation of patients with varying profiles of positive criteria, as well as noncriteria aPLs, may help understand the interval between serological positivity and the emergence of clinical manifestations.

\section{Conclusions}

Recognition of SNAPS patients is critical for clinical management and prevention of potential thrombotic and obstetric adverse events. The non-criteria antiphospholipid antibodies help to identify a considerable portion $(60.9 \%)$ of these patients who otherwise may remain untreated and at clinical risk. aPS/PT IgG and aß2GPI Domain 1 IgG seem to be the most significant risk factors for thrombotic events and pregnancy morbidity, respectively.

\section{Abbreviations}

APS: Antiphospholipid syndrome; aß2GPI : Anti- 32 -glycoprotein; aCL: Anticardiolipin antibodies; LAC: Lupus anticoagulant; SNAPS: Seronegative APS; aPS/PT: Anti-phosphatidylserine/prothrombin antibodies; aß2GPI-D1: AntiB2GPI Domain 1; aPLs: Antiphospholipid antibodies; CAPS: Catastrophic antiphospholipid syndrome; aPT: Anti-prothrombin antibodies; GAPSS: The Global APS Score; LBPA: Anti-lysobisphosphatidic acid; OR: Odds ratio; PAPS: Primary antiphospholipid syndrome; SAPS: Secondary antiphospholipid syndrome; SLE: Systemic lupus erythematosus; ACR: American College of Rheumatology; SLICC: Systemic Lupus International Collaborating Clinics; SS: Sjogren's syndrome; AS: Ankylosing spondylitis; RA: Rheumatoid arthritis; HC: Healthy controls; PM: Pregnancy morbidities; CU: Chemiluminescent units; Cl: Confidence interval; PPV: Positive predictive value; NPV: Negative predictive value; PLR: Positive likelihood ratio; NLR: Negative likelihood ratio; ROC: Receiver operating characteristic curve; AUC: Area under the curve

\section{Acknowledgements}

Not applicable

\section{Honorarium}

Chengde Yang has received an honorarium from Inova Diagnostics.

\section{Author contributions}

$T T L, G L N$, and JFZ carried out the experiment, analysis, and interpretation of data and drafted the manuscript. JYG and LYW contributed to the statistical analysis. QYH helped check the data, JLT, HLL, XBC, JNY, YTS, and YS participated in the collection of clinical data and the collection of the serum samples. CDY, HS, and XFW contribute to revising the intellectual content. All authors read and approved the final manuscript.

\section{Funding}

This work was supported by the National Natural Science Foundation of China (81801602, 81671589, 81871272), Science and Technology Commission of Shanghai Municipality (CN) (17411965100).

\section{Availability of data and materials}

The datasets used and/or analyzed during the current study are available from the corresponding author on reasonable request.

\section{Ethics approval and consent to participate}

The research was performed according to the Declaration of Helsinki and approved by the Institutional Review Broad of Ruijin Hospital (ID: 2016-62), Shanghai Jiaotong University School of Medicine, Shanghai, China. Informed consent was obtained from all individuals included in this study.

\section{Consent for publication \\ Not applicable}

\section{Competing interests}

Gary L. Norman is an employee of Inova Diagnostics, and Jinfeng Zhou is an employee of Werfen China. The funders had no role in the design of the study; in the collection, analyses, or interpretation of data; in the writing of the manuscript; or in the decision to publish the results. The remaining authors report no conflict of interest.

\section{Author details}

${ }^{1}$ Department of Rheumatology and Immunology, Ruijin Hospital, Shanghai Jiao Tong University School of Medicine, No. 197 Ruijin Second Road, Huangpu District, Shanghai 200025, China. ${ }^{2}$ Werfen China, 10 Jiuxianqiao RD. Chaoyang District, Beijing, China. ${ }^{3}$ INOVA Diagnostics, Inc., 9900 Old Grove Road, San Diego, CA 92131, USA. ${ }^{4}$ Department of Laboratory Medicine, Ruijin Hospital, Shanghai Jiao Tong University School of Medicine, Shanghai, China.

Received: 27 August 2019 Accepted: 14 February 2020

Published online: 21 February 2020

\section{References}

1. Hughes GR. Thrombosis, abortion, cerebral disease, and the lupus anticoagulant. Br Med J (Clin Res Ed). 1983;287(6399):1088-9.

2. Wilson WA, Gharavi AE, Koike T, Lockshin MD, Khamashta MA. International consensus statement on preliminary classification criteria for definite antiphospholipid syndrome: report of an international workshop. Arthritis Rheumatol. 1999;42(7):1309-11.

3. Miyakis S, Lockshin MD, Atsumi T, Branch DW, Brey RL, Cervera R, et al. International consensus statement on an update of the classification criteria for definite antiphospholipid syndrome (APS). J Thromb Haemostasis. 2006; 4(2):295-306.

4. Hughes GR, Khamashta MA. Seronegative antiphospholipid syndrome. Ann Rheum Dis. 2003;62(12):1127.

5. Rodriguez-Garcia JL, Bertolaccini ML, Cuadrado MJ, Sanna G, Ateka-Barrutia O, Khamashta MA. Clinical manifestations of antiphospholipid syndrome (APS) with and without antiphospholipid antibodies (the so-called 'seronegative APS'). Ann Rheum Dis. 2012;71(2):242-4.

6. Lazurova I, Macejova Z, Tomkova Z, Remenar F, Boor A, Lazur J, et al. Severe limb necrosis: primary thrombotic microangiopathy or "seronegative" catastrophic antiphospholipid syndrome? A diagnostic dilemma. Clin Rheumatol. 2007;26(10):1737-40.

7. Joalland F, de Boysson H, Darnige L, Johnson A, Jeanjean C, Cheze S, et al. Seronegative antiphospholipid syndrome, catastrophic syndrome, new anticoagulants: learning from a difficult case report. Rev Med Interne. 2014; 35(11):752-6.

8. Losonczy LI, Johnson B, Sidhu J, Li J, Feeney CM. Seronegative catastrophic antiphospholipid syndrome in a young female presenting with a headache. J Emerg Med. 2015;48(3):294-7.

9. Nayfe R, Uthman I, Aoun J, Saad Aldin E, Merashli M, Khamashta MA. Seronegative antiphospholipid syndrome. Rheumatology (Oxford). 2013; 52(8):1358-67.

10. Sciascia S, Sanna G, Murru V, Roccatello D, Khamashta MA, Bertolaccini ML. Anti-prothrombin (aPT) and anti-phosphatidylserine/prothrombin (aPS/PT) antibodies and the risk of thrombosis in the antiphospholipid syndrome. A systematic review. Thromb Haemost. 2014;111(2):354-64. 
11. Hoxha A, Mattia E, Tonello M, Grava C, Pengo V, Ruffatti A. Antiphosphatidylserine/prothrombin antibodies as biomarkers to identify severe primary antiphospholipid syndrome. Clin Chem Lab Med. 2017;55(6): 890-8.

12. Pregnolato F, Chighizola CB, Encabo S, Shums Z, Norman GL, Tripodi A, et al. Anti-phosphatidylserine/prothrombin antibodies: an additional diagnostic marker for APS? Immunol Res. 2013;56(2-3):432-8.

13. Sciascia S, Radin M, Cecchi I, Rubini E, Scotta A, Rolla R, et al. Reliability of lupus anticoagulant and anti-phosphatidylserine/prothrombin autoantibodies in antiphospholipid syndrome: a multicenter study. Front Immunol. 2019;10:376.

14. Shi H, Zheng H, Yin YF, Hu QY, Teng JL, Sun Y, et al. Antiphosphatidylserine/ prothrombin antibodies (aPS/PT) as potential diagnostic markers and risk predictors of venous thrombosis and obstetric complications in antiphospholipid syndrome. Clin Chem Lab Med. 2018;56(4):614-24.

15. Sciascia S, Bertolaccini ML. Thrombotic risk assessment in APS: the Global APS Score (GAPSS). Lupus. 2014;23(12):1286-7.

16. Hammel M, Kriechbaum M, Gries A, Kostner GM, Laggner P, Prassl R. Solution structure of human and bovine beta (2)-glycoprotein I revealed by small-angle X-ray scattering. J Mol Biol. 2002;321(1):85-97.

17. Agar C, van Os GM, Morgelin M, Sprenger RR, Marquart JA, Urbanus RT, et al. Beta2-glycoprotein I can exist in 2 conformations: implications for our understanding of the antiphospholipid syndrome. Blood. 2010;116(8):1336-43.

18. Shemer A, Willis R, Gonzalez EB, Romay-Penabad Z, Shovman O, Shoenfeld $Y$, et al. Oral administration of domain-I of beta-2glycoprotein-I induces immunological tolerance in experimental murine antiphospholipid syndrome. J Autoimmun. 2019;99:98-103.

19. Andreoli L, Chighizola CB, Nalli C, Gerosa M, Borghi MO, Pregnolato F, et al. Clinical characterization of antiphospholipid syndrome by detection of $\lg G$ antibodies against beta2 -glycoprotein i domain 1 and domain 4/5: ratio of anti-domain 1 to anti-domain 4/5 as a useful new biomarker for antiphospholipid syndrome. Arthritis Rheumat (Hoboken). 2015;67(8):2196204.

20. Perez D, Tincani A, Serrano M, Shoenfeld Y, Serrano A. Antiphospholipid syndrome and IgA anti-beta2-glycoprotein I antibodies: when Cinderella becomes a princess. Lupus. 2018;27(2):177-8.

21. Lakos G, Favaloro EJ, Harris EN, Meroni PL, Tincani A, Wong RC, et al. International consensus guidelines on anticardiolipin and anti-beta2glycoprotein I testing: report from the 13th International Congress on Antiphospholipid Antibodies. Arthritis Rheum. 2012;64(1):1-10.

22. Ruiz-Garcia R, Serrano M, Martinez-Flores JA, Mora S, Morillas L, Martin-Mola MA, et al. Isolated IgA anti- beta2 glycoprotein I antibodies in patients with clinical criteria for antiphospholipid syndrome. J Immunol Res. 2014;2014: 704395

23. Arachchillage DR, Efthymiou M, Mackie IJ, Lawrie AS, Machin SJ, Cohen H. Anti-protein $C$ antibodies are associated with resistance to endogenous protein $\mathrm{C}$ activation and a severe thrombotic phenotype in antiphospholipid syndrome. J Thromb Haemostasis. 2014;12(11):1801-9.

24. Sorice M, Griggi T, Circella A, Lenti L, Arcieri P, Domenico di Nucci G, et al. Protein S antibodies in acquired protein S deficiencies. Blood. 1994;83(8): 2383-4.

25. Ortona E, Capozzi A, Colasanti T, Conti F, Alessandri C, Longo A, et al. Vimentin/cardiolipin complex as a new antigenic target of the antiphospholipid syndrome. Blood. 2010;116(16):2960-7.

26. Sorice M, Ferro D, Misasi R, Pittoni V, Longo A, Circella A, et al. Evidence for anticoagulant activity and beta2-GPI accumulation in late endosomes of endothelial cells induced by anti-LBPA antibodies. Thromb Haemost. 2002; 87(4):735-41.

27. Conti F, Alessandri C, Spinelli FR, Capozzi A, Martinelli F, Recalchi S, et al. TLC immunostaining for detection of "antiphospholipid" antibodies. Methods Mol Biol (Clifton). 2014;1134:95-101.

28. Alessandri C, Sorice M, Bombardieri M, Conigliaro P, Longo A, Garofalo T, et al. Antiphospholipid reactivity against cardiolipin metabolites occurring during endothelial cell apoptosis. Arthritis Res Ther. 2006;8(6):R180.

29. Forastiero RR, Martinuzzo ME, Carreras LO. Binding properties of antibodies to prothrombin and beta2-glycoprotein I (beta2-GPI) assayed by ELISA and dot blot. Clin Exp Immunol. 1999;118(3):480-6.

30. Persijn L, Decavele AS, Schouwers S, Devreese K. Evaluation of a new set of automated chemiluminescense assays for anticardiolipin and anti-beta2glycoprotein I antibodies in the laboratory diagnosis of the antiphospholipid syndrome. Thromb Res. 2011;128(6):565-9.
31. Abreu MM, Danowski A, Wahl DG, Amigo MC, Tektonidou M, Pacheco MS, et al. The relevance of "non-criteria" clinical manifestations of antiphospholipid syndrome: 14th International Congress on Antiphospholipid Antibodies Technical Task Force Report on Antiphospholipid Syndrome Clinical Features. Autoimmun Rev. 2015;14(5): 401-14.

32. Hochberg MC. Updating the American College of Rheumatology revised criteria for the classification of systemic lupus erythematosus. Arthritis Rheum. 1997:40(9):1725

33. Petri M, Orbai AM, Alarcon GS, Gordon C, Merrill JT, Fortin PR, et al. Derivation and validation of the Systemic Lupus International Collaborating Clinics classification criteria for systemic lupus erythematosus. Arthritis Rheum. 2012;64(8):2677-86.

34. Shiboski CH, Shiboski SC, Seror R, Criswell LA, Labetoulle M, Lietman TM, et al. 2016 American College of Rheumatology/European League Against Rheumatism classification criteria for primary Sjogren's syndrome: a consensus and data-driven methodology involving three international patient cohorts. Ann Rheum Dis. 2017;76(1):9-16.

35. van der Linden S, Valkenburg HA, Cats A. Evaluation of diagnostic criteria for ankylosing spondylitis. A proposal for modification of the New York criteria. Arthritis Rheum. 1984;27(4):361-8.

36. Aletaha D, Neogi T, Silman AJ, Funovits J, Felson DT, Bingham CO 3rd, et al. 2010 rheumatoid arthritis classification criteria: an American College of Rheumatology/European League Against Rheumatism collaborative initiative. Ann Rheum Dis. 2010;69(9):1580-8.

37. Meijide H, Sciascia S, Sanna G, Khamashta MA, Bertolaccini ML. The clinical relevance of IgA anticardiolipin and IgA anti-beta2 glycoprotein I antiphospholipid antibodies: a systematic review. Autoimmun Rev. 2013; 12(3):421-5.

38. Martinez-Flores JA, Serrano M, Alfaro J, Mora S, Paz-Artal E, Morales JM, et al. Heterogeneity between diagnostic tests for IgA anti-beta2 glycoprotein I: explaining the controversy in studies of association with vascular pathology. Anal Chem. 2013:85(24):12093-8.

39. Chighizola CB, Pregnolato F, Andreoli L, Bodio C, Cesana L, Comerio C, et al. Beyond thrombosis: anti-beta2GPI domain 1 antibodies identify late pregnancy morbidity in anti-phospholipid syndrome. J Autoimmun. 2018;90: 76-83.

40. Zohoury N, Bertolaccini ML, Rodriguez-Garcia JL, Shums Z, Ateka-Barrutia O, Sorice $\mathrm{M}$, et al. Closing the serological gap in the antiphospholipid syndrome: the value of "non-criteria" antiphospholipid antibodies. J Rheumatol. 2017:44(11):1597-602.

41. Litvinova E, Darnige L, Kirilovsky A, Burnel Y, de Luna G, Dragon-Durey MA. Prevalence and significance of non-conventional antiphospholipid antibodies in patients with clinical APS criteria. Front Immunol. 2018:9:2971.

42. Seisdedos L, Munoz-Rodriguez FJ, Cervera R, Font J, Ingelmo M. Primary antiphospholipid syndrome evolving into systemic lupus erythematosus. Lupus. 1997;6(3):285-6.

\section{Publisher's Note}

Springer Nature remains neutral with regard to jurisdictional claims in published maps and institutional affiliations.

Ready to submit your research? Choose BMC and benefit from:

- fast, convenient online submission

- thorough peer review by experienced researchers in your field

- rapid publication on acceptance

- support for research data, including large and complex data types

- gold Open Access which fosters wider collaboration and increased citations

- maximum visibility for your research: over $100 \mathrm{M}$ website views per year

At $\mathrm{BMC}$, research is always in progress.

Learn more biomedcentral.com/submission 